Review Article COVID-19 - Special Issue

\title{
Extreme phenotypes approach to investigate host genetics and COVID-19 outcomes
}

\author{
Michel Satya Naslavsky ${ }^{1}$ (D), Mateus Vidigal ${ }^{1}$, Larissa do Rêgo Barros Matos ${ }^{1}$, Vivian Romanholi Cória ${ }^{1}$, \\ Pedro Benedito Batista Junior ${ }^{2}$, Álvaro Razuk ${ }^{2}$, Paulo Hilário Nascimento Saldiva ${ }^{3}$, Marisa Dolhnikoff ${ }^{3}$, \\ Laire Schidlowski ${ }^{4}$, Carolina Prando ${ }^{4}$, Edécio Cunha-Neto ${ }^{5}$, Antonio Condino-Neto ${ }^{6}$, \\ Maria Rita Passos-Bueno ${ }^{1}$ and Mayana Zatz ${ }^{1}$ \\ ${ }^{1}$ Universidade de São Paulo, Instituto de Biociências, Departamento de Genética e Biologia Evolutiva, \\ Centro de Pesquisa sobre o Genoma Humano e Células-Tronco, São Paulo, SP, Brazil. \\ ${ }^{2}$ Instituto Prevent Senior, São Paulo, SP, Brazil. \\ ${ }^{3}$ Faculdade de Medicina da Universidade de São Paulo (FMUSP), Departamento de Patologia, \\ São Paulo, SP, Brazil. \\ ${ }^{4}$ Instituto de Pesquisa Pelé Pequeno Príncipe, Faculdades Pequeno Príncipe, Hospital Pequeno Príncipe, \\ Curitiba, PR, Brazil. \\ ${ }^{5}$ Hospital das Clínicas da Faculdade de Medicina da Universidade de São Paulo, Instituto do Coração, \\ São Paulo, SP, Brazil. \\ ${ }^{6}$ Universidade de São Paulo, Instituto de Ciências Biomédicas, Laboratório de Imunologia Humana, \\ São Paulo, Brazil.
}

\begin{abstract}
COVID-19 comprises clinical outcomes of SARS-CoV-2 infection and is highly heterogeneous, ranging from asymptomatic individuals to deceased young adults without comorbidities. There is growing evidence that host genetics play an important role in COVID-19 severity, including inborn errors of immunity, age-related inflammation and immunosenescence. Here we present a brief review on the known order of events from infection to severe system-wide disturbance due to COVID-19 and summarize potential candidate genes and pathways. Finally, we propose a strategy of subject's ascertainment based on phenotypic extremes to take part in genomic studies and elucidate intrinsic risk factors involved in COVID-19 severe outcomes.
\end{abstract}

Keywords: COVID-19, host genetics, extreme phenotypes, infectious disease.

Received: August 20, 2020; Accepted: January 18, 2021.

Worldwide populations were significantly affected in 2020 due to the SARS-CoV-2 virus outbreak and individual's health was heterogeneously affected regarding age, sex and comorbidities by the clinical manifestation of the infection (COVID-19). Up to 17\% are severe cases of COVID-19 leading to hospitalization and ultimately causing up to $6 \%$ of death. Some patients require intensive care units (ICU), which faces overflowing occupancy during pandemic peaks (Garg et al., 2020; Griffin, 2020). This balance between the relative and absolute number of individuals with severe symptoms and corresponding distribution of healthcare capacity is critical to prevent a sanitary crisis (Vergano et al., 2020). When ICUs run in full occupancy, mild to severe cases that would eventually be treated and dismissed may progress and lead to worse outcomes than expected under normal occupancy conditions, not to mention overwhelming healthcare teams that care for patients with other conditions (Rosenbaum and Malina, 2020), exposure of staff, and disrupting protective equipment supply chain (Cavallo et al., 2020). Therefore,

Send correspondence to Michel Satya Naslavsky. Universidade de São Paulo, Instituto de Biociências, Departamento de Genética e Biologia Evolutiva, Centro de Pesquisa sobre o Genoma Humano e CélulasTronco, 05508-090, São Paulo, SP, Brazil. E-mail: mnaslavsky@usp.br. predicting the proportion of individuals at higher risk of severity could improve healthcare management decisions and budget allocation by implementation of evidence-based public policies. Risk segmentation can be observed empirically by stratification of individuals based in age, sex, previous comorbidities, and markers (Zheng et al., 2020). However, many risk factors are prevalent and the extent of population-based risk could be more precisely achieved by profiling individual's genomic variability that is associated to increased risk, in a similar fashion of estimating carriers of pathogenic alleles linked to recessive disorders (Zhang et al., 2019) or newborn screening for treatable monogenic conditions (Kelly et al., 2016).

Even though comorbidities are undoubtedly risk factors in COVID-19 severe outcomes, the fact that a proportion of individuals with a healthy status, unaffected by prevalent chronic disorders associated with COVID-19 severity or young patients are progressing to severe complications, suggest a role of genetic susceptibility (Zhang et al., 2020). Such risk profiles are likely to be concealed in the absence of challenging triggers, such as an infection. Indeed, inborn errors of immunity or immunodeficiencies that segregate in families are rare clinical phenotypes caused by highly penetrant variants in over 400 genes (Tangye et al., 2020; Zhang et al., 2020). 
In theory, even if combined, such monogenic manifestations are likely to explain, at most, a fraction of cases, of yet unknown magnitude.

Given the rate of severe outcomes across the population strata, regardless of ancestries and admixture, and the wide heterogeneity of genes and pathways implicated in hostpathogen interactions, it is reasonable to assume a multifactorial mode of inheritance for susceptibility to COVID-19 progression. Such model, however, implies challenges to study design and analytical choices, since there is no prior knowledge of which cases might be explained by rare Mendelian inherited conditions or by a polygenic profile resulting from rare and common variants with individually small to moderate effect sizes. Modulation of risk by indirect genetic factors (e.g. causal factors of comorbidities) and environmental factors, such as exposure to variable viral loads (He et al., 2020) or co-infections that may overwhelm shared systems with SARSCoV-2 (Bradbury et al., 2020), demonstrate the complexity in pinpointing susceptibility drivers (Figure 1).

The wide variability of symptoms in individuals infected by SARS-COV-2 allows to hypothesize candidate genes and pathways that might play a role in risk of (and alternatively, in protection against) severity progression of COVID-19, harboring variation that alters function and/or expression with heterogeneous contribution to the combined individual profile. The X-linked $A C E 2$ gene encodes the Angiotensin-converting enzyme 2, which is expressed in many organs, including nasal and upper airway mucosa, interacts with SARS-CoV-2 spike proteins, followed by TMPRSS2-mediated spike processing and facilitation of viral entry into host cells (Wu and Zheng, 2020; Yan et al., 2020). SLC6A19, although not co-expressed in the upper respiratory tract (Wu and Zheng, 2020), is mainly expressed in enterocytes and antagonizes TMPRSS2, and may play a role in gastrointestinal virion intake. TMPRSS2 is indeed key for viral fusion and considered a candidate for drug targets reducing viral entry (Catanzaro et al., 2020).

It is plausible that coding and regulatory variants in the genes encoding products associated to direct viral entry might influence susceptibility and protection against the first step of infection. It was hypothesized that the higher incidence of COVID-19 in men compared to women could be associated to X-linked factors (Gagliardi et al., 2020). There is evidence for higher ACE2 expression in lungs in patients with comorbidities (Pinto et al., 2020), but sex-specific data are still not available and the balance between hemizygous expression in men and local $\mathrm{X}$-inactivation in women require further investigation. Recently, a sequencing study of Italian patients has suggested common and rare coding variants in ACE2 enriched in patients when compared to controls (Benetti et al., 2020), although a larger number of samples would be ideal to provide supporting evidence. Other host factors involved in formation and activation of the replicasetranscription complex (RTC) are likely to influence success of initial cellular infection cycles (Baric et al., 2008), among which some were detected with an in vitro proteomics approach (V'kovski et al., 2019).

Once viral particles start replicating in host cells, activation of innate and adaptive immune response is expected under physiological conditions. Activating the innate immune response with exacerbated release of proinflammatory cytokines and chemokines (known as 'cytokine storm') has been associated with disease severity. This terminology is forgiving, since it accepts heterogeneous patterns of changes in mediator levels in attempt of fighting infection, ultimately being deleterious to host cells themselves (Tisoncik et al., 2012). During the acute response, interleukin-6 (IL-6) is key and has been consistently observed in high levels among patients with severe COVID-19 outcomes and generic hyperinflammatory

\section{SARS-CoV-2 intrinsic factors}

Virulence (Viral genome);

Source of viral loads.

2. Orthogonal factors

Other infections;

Transmission media;

Exposure to viral loads;

Viral entry pathway.

3. Invasion in upper respiratory tract

Entry cells: receptors ACE2

TMPRSS2, SLC6A19;

RTC host factors;

Salivary constitution;

Prior conditions (Asthma, COPD):

Somatic transformation;

Oral microbiota

4. Innate immune response

Inflammation markers (Cytokine storm:

IL-6, TNF $\alpha$;

Type I/III Interferon Response

Pre-infection inflammatory syndromes

or comorbidities:

MIS-C, Inflammaging, Metabolic

syndromes

NLRP3-mediated pyroptosis;

Germline HLA variability

\section{1}
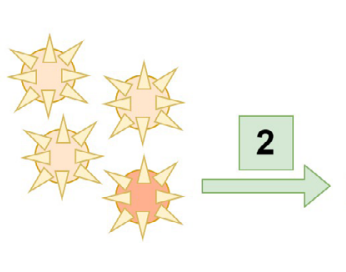

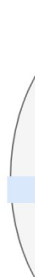

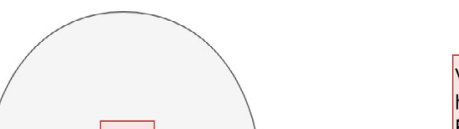

5. Pulmonary tract Variability on respiratory function and hypoxemia;

Radiographical findings: Diffuse alveolar damage; Microthrombosis and endothelial injury:

6. Adaptive immune response Infiltration of T-cells on lungs: Lymphopenia; Lymphopenia; Somatic immunosenescence

7. Systemic tissue damage

Coagulopathy leading to coronary affections, renal failure and cerebrovascular events

Figure 1 - Factors driving SARS-CoV-2 infection and severity of COVID-19 outcomes. Non-host factors (green) and intrinsic host factors (pink) in seven hypothetical steps that may vary and result in different outcomes. 
phenotype (Sinha et al., 2020). Many pathways leading to viral stimulation of innate response and inflammation might play a role in SARS-CoV-2. The canonical and non-canonical inflammasome NLRP3-mediated pathways are candidates since they are sensitive to single stranded RNA viruses and induce pyroptosis fate on host cells (Hayward et al., 2018), which in turn releases pro-inflammatory factors systemwide (Yap et al., 2020). Indeed, the NLRP3 inflammasome is activated in response to SARS-CoV-2 infection, and inflammasome-derived products in sera were found to be correlated with COVID-19 severity (Rodrigues et al., 2020).

The primary source of the exacerbated inflammation itself is still an open problem. If prior chronic inflammation is observed, would it drive the infection-mediated proinflammatory response to reach a certain systemic threshold? There are three major axes of empirical evidence that might support this hypothesis. First, children may be predisposed to hyperinflammatory responses. Cases of multisystem inflammatory syndrome in children (MIS-C) are being observed in severe COVID-19 worldwide (Dufort et al., 2020; Feldstein et al., 2020), an otherwise rare condition that is similar to Kawasaki syndrome, where children manifest inflammation in various systems. As an outcome, cardiovascular dysfunctions are observed, along with elevated levels of C-reactive protein (CRP), D-dimer and troponin, most of which are also observed in high levels in aged patients with severe COVID-19 (Li Q et al., 2020a; Zhou et al., 2020).

Second, the elevated baseline inflammatory status in elderly ('Inflammaging') is associated to frailty, morbidity and mortality regardless of infection (chronic low-grade sterile inflammation). Several factors are associated to this condition, but, at large, senescent cells, including lymphocytes, that secrete cytokines, chemokines and other pro-inflammatory appear to be involved in this phenotype (Akbar and Gilroy, 2020). Although somatic variation is likely to play a role in immunosenescence, it is observed that the distribution of age-related release of pro-inflammatory molecules is variable and long-lived individuals may escape the 'inflammaging' phenotype (Fulop et al., 2017).

The last empirical evidence lies in the risk of comorbidities associated with severe COVID-19. Since the beginning of the pandemic, it has been reported that hypertension, obesity and diabetes (Guan et al., 2020; Richardson et al., 2020) are risk factors for critical COVID-19 outcomes, including death. Low-grade chronic inflammation is a common feature of obesity and metabolic syndromes, and its role in increasing the risk of complications due to SARS-CoV-2 infection cannot be excluded, deserving further investigation (Chiappetta et al., 2020).

Regarding adaptive immunity, decrease of lymphocyte counts and sub-population distribution have also been correlated with worse COVID-19 progression, specifically reduced proportions of CD4+ and CD8+ as predictors of mortality and organ damage (Li D et al., 2020b). There are potential causes for lymphopenia to be considered, including direct infection of lymphocytes, which require further confirmation due to the fact that these cells are likely to be ACE2-negative (Hamming et al., 2004; Xu et al., 2020). In addition, pathological dissections in post-mortem samples should confirm damage to lymphatic organs such as thymus and spleen, leading to lymphocytic dysfunction (Tan et al., 2020). The above mentioned disruption of cytokines' release, such as IL-6 and especially tumor necrosis factor (TNF) $\alpha$, could down-regulate differentiation and may impact proliferation of exhausted $\mathrm{T}$ lymphocytes (Moro-Garcia et al., 2018). Although this is still speculative, immunotherapy trials for COVID-19 by blocking immune checkpoints were suggested (Pickles et al., 2020) and require further confirmation (Luo et al., 2020). Lymphocytes can also be inhibited by circulating lactic acid (Chhetri et al., 2020). Although considering metabolic acidosis as a risk factor during COVID-19 elevated cytokine release is still hypothetical, circulating lactate dehydrogenase (LDH) is consistently observed in patients with severe outcomes (Han et al., 2020; Lu et al., 2020) and might reflect system-wide cell death through apoptosis and pyroptosis (Rayamajhi et al., 2013). Lastly, immunosenescence can be accelerated in elderly due to accumulation of somatic mutations (Zhang, Dong et al., 2019).

The major mechanism of immune evasion of coronaviruses including SARS-CoV-2 is interference with multiple nonstructural and accessory proteins of coronaviruses in the production of and response to type I (Hadjadj et al., 2020) and type III interferons (Yuen et al., 2020). Combined with the association of an early innate antiviral interferon response with mild (Park and Iwasaki, 2020), it is possible to anticipate that variants in genes associated with this pathway may render carriers refractory to productive COVID-19 infection.

Once the infection is established on alveoli, progression to pneumonia, breathing difficulties, and hypoxemia lead patients to mechanical ventilatory support. Qualitative and quantitative thresholds on chest radiographic findings, mostly peripheral lung ground-glass opacities, are being used at the healthcare front as COVID-19 probable classification prior to RT-PCR confirmation, since clinical worsening can be steep (Shi et al., 2020). Variability is observed among asymptomatic: even before classical symptoms appear, a proportion of individuals that tested positive had imaging findings (Meng et al., 2020). Interestingly, lung abnormalities could be found in a case that wasn't primarily aware of COVID-19 (Barajas et al., 2020), although it is still early to estimate the rate of incidental findings.

There is, therefore, heterogeneity regarding the correlation of symptoms, lung damage and hypoxemia, which in turn can require interventions such as invasive mechanical ventilation. In an attempt to understand the most severe outcomes, autopsy studies were initiated in patients who died of COVID-19, in order to investigate pathological changes in several organs. Postmortem lung analysis identified diffuse alveolar damage in different stages of tissue injury and repair by fibroproliferation, and a high incidence of pulmonary embolism, which can account for up to a third of death causes (Deshpande, 2020; Duarte-Neto et al., 2020). Microthrombosis in the alveolar tissue has been detected in up to $80 \%$ of the cases (Dolhnikoff et al 2020; Duarte-Neto et al., 2020). Further investigation revealed infiltration of T-cells, severe endothelial injury, presence of intracellular virus and cell membrane disruption, with evidence of intussusceptive angiogenesis, suggesting a compensatory response (Ackermann et al., 2020). 
Overall, thromboembolic events in the lungs or elsewhere such as heart, kidneys and brain are likely causes of death in severe cases, indicating a probable role for vascular phenotypes, mainly clot formation and distribution (Wadman, 2020). Coagulopathy is supported by consistent findings of elevated D-dimer levels, which in combination with CRP levels, are predictive of critical illness and death from admission date and beyond (Al-Samkari et al., 2020). Therefore, recovered survivors from severe complications due to COVID-19 are likely to have overcome most vascular phenotypes but may have persistent symptoms due to damaged lungs (Carfî et al., 2020) and neurological features, particularly encephalomyelitis (Paterson et al., 2020).

The sequence of observed events and description of general endophenotypes from infection to death or recovery, along the spectrum of COVID-19 progression raises concerns on how to define and select groups of individuals that would capture underlying causality signals, particularly in complex phenotypes with likely multifactorial inheritance. It has been previously proposed that extreme phenotypes might improve the ability of identifying variants and genes ranked with larger effect sizes (Pérez-Gracia et al., 2010; Peloso et al., 2016). This can be achieved by selecting groups with welldefined phenotypes from the extremes of the distribution, and performing rare-variant association studies such as burden analyses, where collapsing variants into biologically relevant sets, usually genes and pathways, provide signals of association even in smaller sample sizes (Lee et al., 2014). A similar collection strategy in COVID-19 has been proposed by expert groups in immunodeficiency research (Casanova et al., 2020).
Considering the extent of SARS-CoV-2 infection and COVID-19 heterogeneity in outcomes worldwide, retrospective classification and re-grouping is already being applied by strategies of meta-analyses. Regarding power estimates when using extreme sampling as an optimization strategy, Peloso et al. (2016) performed simulations using real data of HDL-C distributions and a combination of rare and common variants in $A B C A 1$ gene and provided evidence that smaller sample sizes are required to achieve comparable power on common variants with reduced effect sizes, trading off with some level of overestimation of the proportion of functional variants. If selection threshold is stricter, likelihood of power gains due to extreme selection increases. It has been advocated that since rare variants have a wider effect size range, sequencing studies with burden strategies can benefit from extreme sampling (Peloso et al., 2016; Barnett et al., 2013).

We propose to enrich groups of individuals that have severe lethal outcome with others that remain asymptomatic, ideally against the odds of commonly observed features, such as age and distribution of comorbidities (Figure 2).

Considering the phase of contamination itself to be partially explained by genetics, we have initiated a collection of exposed unaffected individuals. Since controlling for exposure is challenging, our rationale involved the collection of couples or individuals from the same household that are discordant for symptoms and COVID-19 confirmation (one positive and one negative). Special attention will be given to concordance or discordance in twins, monozygotic as compared to dizygotic twins, even though it is unlikely to identify adult twins coliving (fulfilment of exposure control). Asymptomatic exposed

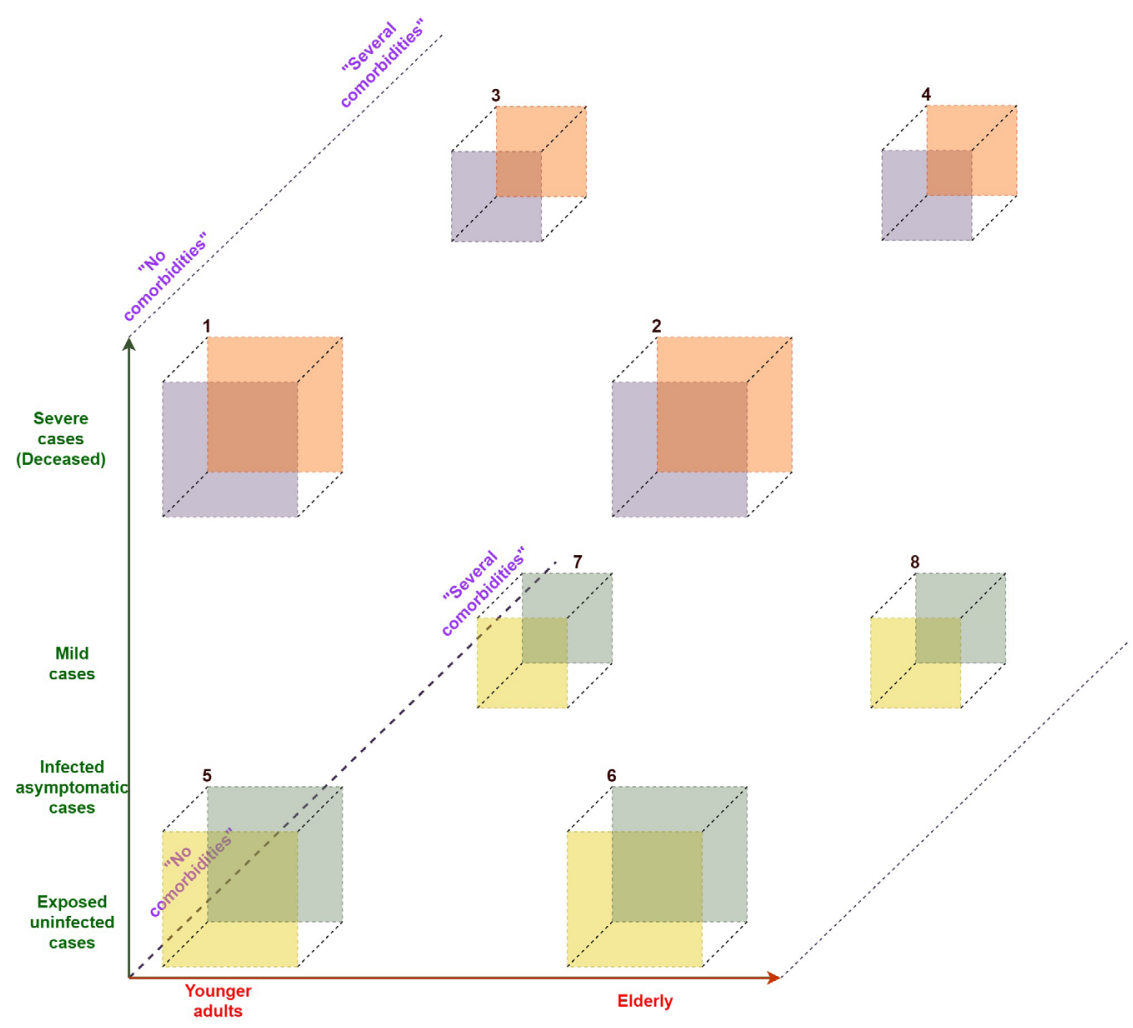

Figure 2 - Hypothetical distribution of extreme COVID-19 phenotypes per generalized risk groups. Groups 1-4 comprise the most susceptible ends. Group 1 opposes to Group 8, from most susceptible to most resistant. Individuals below 1-4 groups are expected to have severe outcomes but not leading to death, and compose a 'resilient' phenotype towards the corner below group 4 (elderly, with several comorbidities that were recovered). 
individuals who test negative for viremia and antibodies are named 'Resistant'. Since exposure levels might determine an infection event after initial sampling and that the viral strains are likely to be the same within each pair of individuals, additional confirmation of negative infection after periods of time will support 'Resistant' phenotype, or alternatively reclassify these individuals as 'Susceptible' under a different context.

As a second group, we began collecting samples from patients who died of COVID-19 and underwent autopsy procedure classified as a 'Susceptible' group. Retrospective collection of presence of comorbidities and estimating evidences of ante-mortem versus post-mortem vascular pathologies may provide a glimpse of prior risk and injuries due to SARS-CoV-2 infection itself.

Third, a collection of recovered nonagenarians and centenarians might provide insights on compensatory effects of system-wide resilience (named 'Resilience' group), which are likely to result from a combination of effective immune response, reduced levels of inflammation and protection from coagulopathies. In this stage, we aim at collecting DNA, RNA and peripheral blood mononuclear cells from individuals admitted to a hospital and recollecting after discharge, providing additional molecular phenotypes to improve filtering variants within genes and pathways observed to be altered.

This ongoing project (IRB approval, CEP IB-USP CAAE 34786620.2.0000.5464) has to date collected clinical data and biological samples from 380 individuals, most of which have been already whole-exome sequenced, RNA and vials of peripheral blood mononucleate cells (PBMCs) from most participants stored for downstream functional analyses.

Genome-wide association studies (GWAS) with casecontrol design in COVID-19 started to yield results in Spanish and Italian individuals. They have detected association signals in a locus at $3 \mathrm{p} 21.31$ comprising interesting candidates such as genes encoding SIT1, an interactor with ACE2, and CXCR6, a regulator of memory CD8 T cells (Ellinghaus et al., 2020). ABO locus was also detected, overlapping with a clinical suggestion of specific blood types being enriched in severe groups, although this is still controversial (Latz et al., 2020; Wu et al., 2020). The authors stress the need for ascertainment of controls, since dynamics of infection and variable symptomatology might compromise stratification.

Traditional GWAS using common variants may not capture the strongest association signals, since genetic heterogeneity across groups of patients and controls may promote diverse and complex genomic architectures. Including rare variation obtained by sequencing whole exomes or, ideally, whole genomes, followed by burden analyses where rare variants are collapsed per gene or pathway might increase the ability to detect signals (Lanktree et al., 2010; Peloso et al., 2016; Xu et al., 2018). Selection and characterization of extreme phenotypes in response to HIV-1 infection led to discovery of the 32-base pair deletion in the chemokine receptor 5 (CCR5A32) (Dean et al., 1996), which helped elucidation of pathways associated to AIDS. Other studies on reduced penetrance within families, that is, extreme phenotypes co-segregating with pathogenic mutation carriers in the same pedigree, can provide clues for modifier genes (Cooper et al., 2013), including those implicated in variable expressivity, for instance compensating the absence of key protein in muscle physiology (Vieira et al., 2015). Differential cellular susceptibility to infection was also verified in twins exposed to Zika virus (Caires-Júnior et al., 2018), suggesting that discordant outcomes can provide insights about the intrinsic factors driving host-pathogen interactions.

We hypothesize that using extreme phenotypes of properly ascertained groups will improve aggregation of variants with larger effect size, which, in combination, will allow polygenic risk stratification. Collaborative initiatives such as the COVID Human Genetic Effort (Zhang et al., 2020) and the COVID-19 Host Genetics Initiative (2020) were organized to systematically aggregate data across different regions worldwide. Since the etiology of COVID-19 host susceptibility and protection is likely to be multifactorial, it is expected that a large number of samples will be needed in order to identify loci that associate with corresponding phenotypes, therefore collaboration is critical. We emphasize the need for including admixed individuals with ancestries that go beyond European, which would not only contribute to reduce health disparities when describing the particularities of severe outcomes on diverse populations, but may provide insights from genes and pathways that harbor specific variants associated to phenotypes of interest such as severe outcomes of COVID-19.

\section{Acknowledgments}

Initial collection funded by Conselho Nacional de Desenvolvimento Científico e Tecnológico (CNPq) 465355/ 2014-5, Fundação de Amparo à Pesquisa do Estado de São Paulo (FAPESP) 2014/50931-3 and 2013/08028-1. Grantee: Mayana Zatz. We acknowledge the research subjects and the team members responsible for sample collections.

\section{Conflict of Interest}

The authors declare that there is no conflict of interest that could be perceived as prejudicial to the impartiality of the reported research.

\section{Authors Contributions}

MSN and MZ conceived the study; MV, LRBM, VR, PHNS, MD, LS, CP are currently collecting, processing and analyzing samples; PBBJ, AR are sample and data providers; PHNS, MD, CP, ECN, ACN are principal investigators of collaborative groups; PBBJ, AR, ECN, ACN, MRPB and MZ provided initial funding for sample collection; MSN wrote the manuscript.

\section{References}

Ackermann M, Verleden SE, Kuehnel M, Haverich A, Welte T, Laenger F, Vanstapel A, Werlein C, Stark H, Tzankov A et al. (2020) Pulmonary vascular endothelialitis, thrombosis, and angiogenesis in COVID-19. N Engl J Med 2:120-128.

Akbar AN and Gilroy DW (2020) Aging immunity may exacerbate COVID-19. Science 6501:256-257.

Al-Samkari H, Karp Leaf RS, Dzik WH, Carlson JCT, Fogerty AE, Waheed A, Goodarzi K, Bendapudi PK, Bornikova L, Gupta $\mathrm{S}$ et al. (2020) COVID-19 and coagulation: bleeding and thrombotic manifestations of SARS-CoV-2 infection. Blood 4:489-500. 
Barajas RF, Rufener G, Starkey J, Duncan T and Fuss C (2020) Asymptomatic COVID-19: What the neuroradiologist needs to know about pulmonary manifestations. Am J Neuroradiol 6:966-968.

Baric RS, Van Hemert MJ, Van Den Worm SHE, Knoops K, Mommaas AM, Gorbalenya AE and Snijder EJ (2008) SARS-Coronavirus replication/transcription complexes are membrane-protected and need a host factor for activity in vitro. PLoS Pathog 4:e1000054.

Barnett IJ, Lee S and Lin X (2013) Detecting rare variant effects using extreme phenotype sampling in sequencing association studies. Genet Epidemiol 37:142-151.

Benetti E, Tita R, Spiga O, Ciolfi A, Birolo G, Bruselles A, Doddato G, Giliberti A, Marconi C, Musacchia F et al. (2020) ACE2 gene variants may underlie interindividual variability and susceptibility to COVID-19 in the Italian population. Eur J Hum Genet 28:1602-1614.

Bradbury RS, Piedrafita D, Greenhill A and Mahanty S (2020) Will helminth co-infection modulate COVID-19 severity in endemic regions? Nat Rev Immunol 6:342-342.

Caires-Júnior LC, Goulart E, Melo US, Araujo BHS, Alvizi L, SoaresSchanoski A, De Oliveira DF, Kobayashi GS, Griesi-Oliveira $\mathrm{K}$, Musso CM et al. (2018) Discordant congenital Zika syndrome twins show differential in vitro viral susceptibility of neural progenitor cells. Nat Commun 9:475.

Carfî A, Bernabei R and Landi F (2020) Persistent symptoms in patients after acute COVID-19. J Amer Med Assoc 6:603.

Casanova JL, Su HC and Effort CHG (2020) A global effort to define the human genetics of protective immunity to SARS-CoV-2 infection. Cell 6:1194-1199.

Catanzaro M, Fagiani F, Racchi M, Corsini E, Govoni S and Lanni C (2020) Immune response in COVID-19: addressing a pharmacological challenge by targeting pathways triggered by SARS-CoV-2. Signal Transduct Target Ther 5:84.

Cavallo JJ, Donoho DA and Forman HP (2020) Hospital capacity and operations in the coronavirus disease 2019 (COVID-19) pandemic-planning for the nth patient. JAMA Health Forum. DOI: 10.1001 /jamahealthforum.2020.0345.

Chhetri S, Khamis F, Pandak N, Al Khalili H, Said E and Petersen E (2020) A fatal case of COVID-19 due to metabolic acidosis following dysregulate inflammatory response (cytokine storm). IDCases:e00829.

Chiappetta S, Sharma AM, Bottino V and Stier C (2020) COVID-19 and the role of chronic inflammation in patients with obesity. Int J Obesity 8:1790-1792.

Cooper DN, Krawczak M, Polychronakos C, Tyler-Smith C and Kehrer-Sawatzki H (2013) Where genotype is not predictive of phenotype: towards an understanding of the molecular basis of reduced penetrance in human inherited disease. Hum Genet 10:1077-1130.

COVID-19 Host Genetics Initiative (2020) The COVID-19 Host Genetics Initiative, a global initiative to elucidate the role of host genetic factors in susceptibility and severity of the SARS-CoV-2 virus pandemic. Eur J Hum Genet 6:715-718.

Dean M, Carrington M, Winkler C, Huttley GA, Smith MW, Allikmets $\mathrm{R}$, Goedert JJ, Buchbinder SP, Vittinghoff E, Gomperts E et al. (1996) Genetic restriction of HIV-1 infection and arogression to AIDS by a deletion allele of the CKR5 structural gene. Science 5283:1856-1862.

Deshpande C (2020) Thromboembolic findings in COVID-19 autopsies: Pulmonary thrombosis or embolism? Ann Intern Med 173:394-395.

Dolhnikoff M, Duarte-Neto AN, Almeida Monteiro RA, Silva LFF, Oliveira EP, Saldiva PHN, Mauad T and Negri EM (2020) Pathological evidence of pulmonary thrombotic phenomena in severe COVID-19. J Thromb Haemost 6:1517-1519.
Duarte-Neto AN, Monteiro RaA, Silva LFF, Malheiros DMaC, Oliveira EP, Theodoro-Filho J, Pinho JRR, Gomes-Gouvêa MS, Salles APM, Oliveira IRS et al. (2020) Pulmonary and systemic involvement in COVID-19 patients assessed with ultrasound-guided minimally invasive autopsy. Histopathology 2:186-197.

Dufort EM, Koumans EH, Chow EJ, Rosenthal EM, Muse A, Rowlands J, Barranco MA, Maxted AM, Rosenberg ES, Easton D et al. (2020) Multisystem inflammatory syndrome in children in New York State. N Engl J Med 4:347-358.

Ellinghaus D, Degenhardt F, Bujanda L, Buti M, Albillos A, Invernizzi P, Fernandez J, Prati D, Baselli G, Asselta R et al. (2020) Genomewide Association Study of severe COVID-19 with respiratory failure. N Engl J Med 383:1522-1534.

Feldstein LR, Rose EB, Horwitz SM, Collins JP, Newhams MM, Son MBF, Newburger JW, Kleinman LC, Heidemann SM, Martin AA et al. (2020) Multisystem inflammatory syndrome in U.S. children and adolescents. N Engl J Med 4:334-346.

Fulop T, Larbi A, Dupuis G, Le Page A, Frost EH, Cohen AA, Witkowski JM and Franceschi C (2017) Immunosenescence and Inflamm-Aging as two sides of the same coin: Friends or foes? Front Immunol 8:1960.

Gagliardi MC, Tieri P, Ortona E and Ruggieri A (2020) ACE2 expression and sex disparity in COVID-19. Cell Death Discov 6:37.

Garg S, Kim L, Whitaker M, O'halloran A, Cummings C, Holstein R, Prill M, Chai SJ, Kirley PD, Alden NB et al. (2020) Hospitalization rates and characteristics of patients hospitalized with laboratory-confirmed Coronavirus Disease 2019 COVID-NET, 14 States, March 1-30, 2020. Morb Mortal Wkly Rep:69:458-464.

Griffin S (2020) COVID-19: Continued outbreaks in care homes risk extending pandemic, say experts. BMJ 369:m2530.

Guan WJ, Liang WH, Zhao Y, Liang HR, Chen ZS, Li YM, Liu XQ, Chen RC, Tang CL, Wang T et al. (2020) Comorbidity and its impact on 1590 patients with COVID-19 in China: a nationwide analysis. Eur Respir J 55:2000547.

Hadjadj J, Yatim N, Barnabei L, Corneau A, Boussier J, Smith N, Pere $\mathrm{H}$, Charbit B, Bondet V, Chenevier-Gobeaux C et al. (2020) Impaired type I interferon activity and inflammatory responses in severe COVID-19 patients. Science 6504:718-724.

Hamming I, Timens W, Bulthuis ML, Lely AT, Navis G and Van Goor $\mathrm{H}$ (2004) Tissue distribution of ACE2 protein, the functional receptor for SARS coronavirus. A first step in understanding SARS pathogenesis. J Pathol 2:631-637.

Han Y, Zhang H, Mu S, Wei W, Jin C, Xue Y, Tong C, Zha Y, Song $\mathrm{Z}$ and $\mathrm{Gu} \mathrm{G}$ (2020) Lactate dehydrogenase, a risk factor of severe COVID-19 patients. medRxiv: 2020.03.24.20040162.

Hayward JA, Mathur A, Ngo C and Man SM (2018) Cytosolic recognition of microbes and pathogens: Inflammasomes in action. Microbiol Mol Biol R 82:e0015-18.

He X, Lau EHY, Wu P, Deng X, Wang J, Hao X, Lau YC, Wong JY, Guan Y, Tan X et al. (2020) Temporal dynamics in viral shedding and transmissibility of COVID-19. Nat Med 5:672-675.

Kelly N, Makarem DC and Wasserstein MP (2016) Screening of newborns for disorders with high benefit-risk ratios should be mandatory. J Law Med Ethics 2:231-240.

Lanktree MB, Hegele RA, Schork NJ and Spence JD (2010) Extremes of unexplained variation as a phenotype. Circ-Cardiovasc Gene 2:215-221.

Latz CA, Decarlo C, Boitano L, Png CYM, Patell R, Conrad MF, Eagleton M and Dua A (2020) Blood type and outcomes in patients with COVID-19. Ann Hematol 9:2113-2118.

Lee S, Abecasis Gonçalo r, Boehnke M and Lin X (2014) RareVariant Association Analysis: Study Designs and Statistical Tests. Am J Hum Genet 1:5-23. 
Li D, Chen Y, Liu H, Jia Y, Li F, Wang W, Wu J, Wan Z, Cao Y and Zeng R (2020b) Immune dysfunction leads to mortality and organ injury in patients with COVID-19 in China: insights from ERS-COVID-19 study. Signal Transduct Target Ther 5:62.

Li Q, Ding X, Xia G, Chen H-G, Chen F, Geng Z, Xu L, Lei S, Pan A, Wang L et al. (2020a) Eosinopenia and elevated C-reactive protein facilitate triage of COVID-19 patients in fever clinic: A retrospective case-control study. EClinicalMedicine23:100375.

Lu Y, Sun K, Guo S, Wang J, Li A, Rong X, Wang T, Shang Y, Chang W and Wang S (2020) Early warning indicators of severe COVID-19: A single-center study of cases from Shanghai, China. Front Med (Lausanne) 7:432.

Luo J, Rizvi H, Egger JV, Preeshagul IR, Wolchok JD and Hellmann MD (2020) Impact of PD-1 blockade on severity of COVID-19 in patients with lung cancers. Cancer Discov 8:1121-1128.

Meng H, Xiong R, He R, Lin W, Hao B, Zhang L, Lu Z, Shen X, Fan $\mathrm{T}$, Jiang W et al. (2020) CT imaging and clinical course of asymptomatic cases with COVID-19 pneumonia at admission in Wuhan, China. J Infect 1:e33-e39.

Moro-Garcia MA, Mayo JC, Sainz RM and Alonso-Arias R (2018) Influence of inflammation in the process of $\mathrm{T}$ lymphocyte differentiation: Proliferative, metabolic, and oxidative changes. Front Immunol 9:339.

Park A and Iwasaki A (2020) Type I and Type III interferons induction, signaling, evasion, and application to combat COVID-19. Cell Host Microbe 6:870-878.

Paterson RW, Brown RL, Benjamin L, Nortley R, Wiethoff S, Bharucha T, Jayaseelan DL, Kumar G, Raftopoulos RE, Zambreanu L et al. (2020) The emerging spectrum of COVID-19 neurology: clinical, radiological and laboratory findings. Brain 143:3104-3120.

Peloso GM, Rader DJ, Gabriel S, Kathiresan S, Daly MJ and Neale BM (2016) Phenotypic extremes in rare variant study designs. Eur J Hum Genet 6:924-930

Pérez-Gracia JL, Gúrpide A, Ruiz-Ilundain MG, Alegría CA, Colomer R, García-Foncillas J and Melero Bermejo I (2010) Selection of extreme phenotypes: the role of clinical observation in translational research. Clin Trans Oncol 3:174-180.

Pickles OJ, Lee LYW, Starkey T, Freeman-Mills L, Olsson-Brown A, Cheng V, Hughes DJ, Lee A, Purshouse K and Middleton $\mathrm{G}$ (2020) Immune checkpoint blockade: releasing the breaks or a protective barrier to COVID-19 severe acute respiratory syndrome? Br J Cancer 123:691-693.

Pinto BGG, Oliveira AER, Singh Y, Jimenez L, Gonçalves ANA, Ogava RLT, Creighton R, Schatzmann Peron JP and Nakaya HI (2020) ACE2 expression is increased in the lungs of patients with comorbidities associated with severe COVID-19. J Infect Dis 4:556-563.

Rayamajhi M, Zhang Y and Miao EA (2013) Detection of pyroptosis by measuring released lactate dehydrogenase activity. Methods Mol Biol 1040:85-90.

Richardson S, Hirsch JS, Narasimhan M, Crawford JM, McGinn T, Davidson KW; the Northwell COVID-19 Research Consortium, Barnaby DP, Becker LB, Chelico JD, J et al. (2020) Presenting characteristics, comorbidities, and outcomes among 5700 patients hospitalized with COVID-19 in the New York City area. JAMA 323:2052-2059.

Rodrigues TS, Sa KSG, Ishimoto AY, Becerra A, Oliveira S, Almeida L, Goncalves AV, Perucello DB, Andrade WA, Castro R et al. (2020) Inflammasome activation in COVID-19 patients. medRxiv:2020.08.05.20168872.

Rosenbaum L and Malina D (2020) The untold toll - the pandemic's effects on patients without COVID-19. N Engl J Med 24:23682371.
Shi H, Han X, Jiang N, Cao Y, Alwalid O, Gu J, Fan Y and Zheng C (2020) Radiological findings from 81 patients with COVID-19 pneumonia in Wuhan, China: a descriptive study. Lancet Infect Dis 4:425-434.

Sinha P, Matthay MA and Calfee CS (2020) Is a "Cytokine Storm" relevant to COVID-19? JAMA Int Med 180:1152-1154.

Tan L, Wang Q, Zhang D, Ding J, Huang Q, Tang YQ, Wang Q and Miao $H$ (2020) Lymphopenia predicts disease severity of COVID-19: a descriptive and predictive study. Signal Transduct Target Ther 5:33.

Tangye SG, Al-Herz W, Bousfiha A, Chatila T, CunninghamRundles C, Etzioni A, Franco JL, Holland SM, Klein C, Morio T et al. (2020) Human inborn errors of immunity: 2019 update on the classification from the International Union of Immunological Societies Expert Committee. J Clin Immunol 1:24-64.

Tisoncik JR, Korth MJ, Simmons CP, Farrar J, Martin TR and Katze MG (2012) Into the eye of the cytokine storm. Microbiol Molr Biol R 1:16-32.

V'kovski P, Gerber M, Kelly J, Pfaender S, Ebert N, Braga Lagache S, Simillion C, Portmann J, Stalder H, Gaschen V et al. (2019) Determination of host proteins composing the microenvironment of coronavirus replicase complexes by proximity-labeling. eLife 8:e42037.

Vergano M, Bertolini G, Giannini A, Gristina GR, Livigni S, Mistraletti G, Riccioni L and Petrini F (2020) Clinical ethics recommendations for the allocation of intensive care treatments in exceptional, resource-limited circumstances: the Italian perspective during the COVID-19 epidemic. Crit Care 24:165.

Vieira NM, Elvers I, Alexander MS, Moreira YB, Eran A, Gomes JP, Marshall JL, Karlsson EK, Verjovski-Almeida S, LindbladToh K et al. (2015) Jagged 1 rescues the Duchenne muscular dystrophy phenotype. Cell 5:1204-1213.

Wadman M (2020) How does coronavirus kill? Clinicians trace a ferocious rampage through the body, from brain to toes. Science. https://doi.org/10.1126/science.abc3208.

Wu BB, Gu DZ, Yu JN, Yang J and Wang-Qin S (2020) Association between ABO blood groups and COVID-19 infection, severity and demise: A systematic review and meta-analysis. Infect Genet Evol 84:104485.

Wu C and Zheng M (2020) Single-cell RNA expression profiling shows that ACE2, the putative receptor of COVID-2019, has significant expression in nasal and mouth tissue, and is coexpressed with TMPRSS2 and not co-expressed with SLC6A19 in the tissues (Version 1). BMC Infect Dis Research Square. https://doi.org/10.21203/rs.3.rs-16992/v1.

Xu C, Fang J, Shen H, Wang Y-P, Deng H-W and Hancock J (2018) EPS-LASSO: test for high-dimensional regression under extreme phenotype sampling of continuous traits. Bioinformatics 12:1996-2003

Xu H, Zhong L, Deng J, Peng J, Dan H, Zeng X, Li T and Chen Q (2020) High expression of ACE2 receptor of 2019-nCoV on the epithelial cells of oral mucosa. Int J Oral Sci 1:8.

Yan R, Zhang Y, Li Y, Xia L, Guo Y and Zhou Q (2020) Structural basis for the recognition of SARS-CoV-2 by full-length human ACE2. Science 6485:1444-1448.

Yap JKY, Moriyama M and Iwasaki A (2020) Inflammasomes and pyroptosis as therapeutic targets for COVID-19. J Immunol 2:307-312.

Yuen C-K, Lam J-Y, Wong W-M, Mak L-F, Wang X, Chu H, Cai J-P, Jin D-Y, To KK-W, Chan JF-W et al. (2020) SARS-CoV-2 nsp13, nsp14, nsp15 and orf6 function as potent interferon antagonists. Emerg Microbe Infec 1:1418-1428. 
Zhang L, Bao Y, Riaz M, Tiller J, Liew D, Zhuang X, Amor DJ, Huq A, Petelin L, Nelson M et al. (2019) Population genomic screening of all young adults in a health-care system: a costeffectiveness analysis. Genet Med. 21:1958-1968.

Zhang L, Dong X, Lee M, Maslov AY, Wang T and Vijg J (2019) Single-cell whole-genome sequencing reveals the functional landscape of somatic mutations in B lymphocytes across the human lifespan. Proc Natl Acad Sci U S A 18:9014-9019.

Zhang SY, Zhang Q, Casanova JL, Su HC and Team C (2020) Severe COVID-19 in the young and healthy: monogenic inborn errors of immunity? Nat Rev Immunol 8:455-456.

Zheng Z, Peng F, Xu B, Zhao J, Liu H, Peng J, Li Q, Jiang C, Zhou Y, Liu S et al. (2020) Risk factors of critical \& mortal COVID-19 cases: A systematic literature review and metaanalysis. J Infect 2:e16-e25.
Zhou F, Yu T, Du R, Fan G, Liu Y, Liu Z, Xiang J, Wang Y, Song $\mathrm{B}, \mathrm{Gu} \mathrm{X}$ et al. (2020) Clinical course and risk factors for mortality of adult inpatients with COVID-19 in Wuhan, China: a retrospective cohort study. Lancet 10229:1054-1062.

Associate Editor: Diogo Meyer

License information: This is an open-access article distributed under the terms of the Creative Commons Attribution License (type CC-BY), which permits unrestricted use, distribution and reproduction in any medium, provided the original article is properly cited. 